\title{
Globally exponential stability of a certain neutral differential equation with time-varying delays
}

Panuwat Keadnarmol and Thaned Rojsiraphisal ${ }^{*}$

"Correspondence:

thaned.r@cmu.ac.th Department of Mathematics, Faculty of Science, Chiang Mai University, Chiang Mai, 50200, Thailand

\begin{abstract}
In this paper, an improved globally exponential stability criterion of a certain neutral delayed differential equation with time-varying of the form $\frac{d}{d t}[x(t)+p x(t-\tau(t))]=-a x(t)+b \tanh x(t-\sigma(t))$ has been proposed in the form of linear matrix inequality. We first propose an upper bound of the solution in terms of an exponential function. Then we apply Lyapunov functions, a descriptor form, the Leibniz-Newton formula and radially unboundedness to formulate the sufficient criterion. To show the effectiveness of the proposed criterion, four numerical examples are presented.
\end{abstract}

Keywords: exponential stability; neutral delayed differential equation; linear matrix inequality; Lyapunov functions; descriptor form

\section{Introduction}

In this paper, we investigate the stability of the neutral delayed differential equation (NDE) of the form

$$
\frac{d}{d t}[x(t)+p x(t-\tau(t))]=-a x(t)+b \tanh x(t-\sigma(t)), \quad t \geq 0,
$$

where $a, b$ are positive real constants and $|p|<1$. The delays $\tau(t), \sigma(t)$ are bounded continuous functions defined as $\tau(t):[0, \infty) \rightarrow[0, \tau]$ and $\sigma(t):[0, \infty) \rightarrow[0, \sigma]$ satisfying

$$
0 \leq \tau(t) \leq \tau, \quad 0 \leq \sigma(t) \leq \sigma, \quad \dot{\tau}(t) \leq \delta_{1}, \quad \dot{\sigma}(t) \leq \delta_{2}<1 .
$$

For each solution of equation (1), we assume the initial condition

$$
x_{0}(\theta)=\phi(\theta), \quad \theta \in[-r, 0]
$$

where $r=\max \{\tau, \sigma\}, \phi \in \mathbb{C}([-r, 0] ; \mathbb{R})$.

The NDE (1), consisting of discrete and neutral delays, often appears in scientific and engineering fields such as aircraft, chemical processing, control systems, and biological systems ([1-3] and references therein). It is well known that a small change in delay may destabilize a system $[4,5]$. Therefore, researchers had increased their attention to the study of the stabilization of the system by proposing stability criteria in various forms, commonly

o2014 Keadnarmol and Rojsiraphisal; licensee Springer. This is an Open Access article distributed under the terms of the Creative Commons Attribution License (http://creativecommons.org/licenses/by/2.0), which permits unrestricted use, distribution, and reproduction in any medium, provided the original work is properly cited. 
in the form of a linear matrix inequality (LMI) base. It is also known that the LMI condition can be classified into two categories: delay independent (no information of delay used) and delay dependent (engaged with delay). The latter condition is generally considered as less conservative than the former when the delay is small.

Earlier stability studies of equation (1) had focused on the case of constant delays as in the form

$$
\frac{d}{d t}[x(t)+p x(t-\tau)]=-a x(t)+b \tanh x(t-\sigma), \quad t \geq 0 .
$$

Several techniques such as using a Lyapunov-like theorem method, Lyapunov-Krasovskii functional, integral inequalities, and model transformations have been used to obtain sufficient conditions to ensure stability of the NDE [6-17]. The asymptotic stability of the NDE with constant delays (3) has been discussed in [6, 7, 9-17], while the exponential stability has been studied in $[7,8,16,18]$. There are only three researches $[7,8,18]$ proposing globally exponential stability criteria for the NDE with time-varying delays (1), which can be considered as a more realistic situation since the time delays are usually not constant. However, the criterion proposed in [7] cannot specify the rate of convergence, which may be regarded as only asymptotically stable $[8]$, while criteria proposed in $[8,18]$ are slightly conservative and can be improved.

In this article, we reinvestigate the exponential stability of the NDE (1) with time-varying delays. We also propose an upper bound of solution of the neutral differential equation in terms of an exponential function. Using the descriptor form introduced in [1, 19], a model transformation, Lyapunov-Krasovskii functions, and radially unboundedness, an improved globally exponential stability criterion is formulated in the form of LMI. Finally, four numerical examples are presented to show the effectiveness of the proposed criterion by comparing the upper bounds of the delay $\sigma(t)$ and the parameter $b$ with other existing work.

Notations: Throughout this article, $*$ represents the elements below the main diagonal of a symmetric matrix. The notation $A \geq 0(A>0)$ means $A$ is semi-positive definite (positive definite); $A^{T}$ denotes the transpose of the matrix $A$ and $\|\cdot\|$ denotes the Euclidean norm of given vector or matrix; $\mathbb{R}$ denotes the set of real numbers; $\mathbb{R}^{n}$ denotes the set of $n$-tuples of real numbers; and $\mathbb{R}^{n \times n}$ denotes the set of $n \times n$ matrices whose entries are real.

\section{Preliminaries}

For convenience, we define a new variable $D(t): \equiv x(t)+p x(t-\tau(t))$. Therefore, the NDE (1) can be written as

$$
\dot{D}=\frac{d}{d t}[x(t)+p x(t-\tau(t))]=-a x(t)+b \tanh x(t-\sigma(t)) .
$$

With the new variable above, we now define the descriptor form of equation (1) as follows:

$$
\left\{\begin{array}{l}
\dot{D}=-a x(t)+b \tanh x(t-\sigma(t)) \\
0=-D+x(t)+p x(t-\tau(t)) .
\end{array}\right.
$$


Definition 1 The equilibrium point $x=0$ of the system (1) is exponentially stable if there exist positive real constants $K$ and $\lambda$ such that

$$
\|x(t)\| \leq K e^{-\lambda t} \sup _{-r \leq s \leq 0}\|x(s)\|=K e^{-\lambda t}\left\|x_{0}\right\|_{s}
$$

where $\left\|x_{t}\right\|_{s}=\sup _{-r \leq s \leq 0}\|x(t+s)\|$.

Lemma 2 (Cauchy inequality) For any symmetric positive definite matrix $N \in \mathbb{R}^{n \times n}$ and $x, y \in \mathbb{R}^{n}$, we have

$$
\pm 2 x^{T} y \leq x^{T} N x+y^{T} N^{-1} y .
$$

Proposition 3 Let $M>0, \mu>0,|p|<1$ and $0 \leq \tau(t) \leq \tau$. If $x:[-\tau, \infty) \rightarrow R$, satisfying

$$
\|x(t)\| \leq \sup _{s \in[-\tau, 0]}\|x(s)\|=\left\|x_{0}\right\|_{s}, \quad t \in[-\tau, 0]
$$

and

$$
\|x(t)\| \leq|p|\|x(t-\tau(t))\|+M e^{-\mu t}, \quad t \geq 0
$$

Then there exist positive scalars $\epsilon, m \in\left[0, \frac{-\ln |p|}{\tau}\right]$ such that $|p| e^{\epsilon \tau}<1$ and

$$
\|x(t)\| \leq\left\|x_{0}\right\|_{s} e^{-m t}+\frac{M}{1-|p| e^{\epsilon \tau}} e^{-\epsilon t} \leq N e^{-\varphi t}, \quad t \geq 0,
$$

where $N: \equiv\left\|x_{0}\right\|_{s}+\frac{M}{1-|p| e^{\epsilon \tau}}$ and $\varphi=\min \{m, \epsilon\}$.

Proof Since $|p|<1$ and $0 \leq \tau(t) \leq \tau$, there exist sufficient small scalar $\epsilon, m \in\left[0, \frac{-\ln |p|}{\tau}\right]$ such that $|p| e^{\epsilon \tau}<1$ and $|p| e^{m \tau}<1$. Next we will show that the inequality (6) holds. If $\mu \leq \epsilon$, we choose $\mu=\epsilon$; else if $\mu>\epsilon$, we have $e^{-\mu t} \leq e^{-\epsilon t}$.

For $t=0$, we have

$$
\|x(0)\| \leq|p|\|x(-\tau(0))\|+M \leq|p| \sup _{-\tau \leq s \leq 0}\|x(s)\|+M<\left\|x_{0}\right\|_{s}+\frac{M}{1-|p| e^{\epsilon \tau}} \equiv N .
$$

Thus, the inequality (6) holds for $t=0$.

For $t>0$, suppose the equality (6) fails; i.e. there exists $t^{*}>0$ such that

$$
\begin{aligned}
& \left\|x\left(t^{*}\right)\right\|>\left\|x_{0}\right\|_{s} e^{-m t^{*}}+\frac{M}{1-|p| e^{\epsilon \tau}} e^{-\epsilon t^{*}} \equiv N e^{-\varphi t^{*}} \text { and } \\
& \|x(t)\| \leq\left\|x_{0}\right\|_{s} e^{-m t}+\frac{M}{1-|p| e^{\epsilon \tau}} e^{-\epsilon t} \equiv N e^{-\varphi t}, \quad \text { for all } t \in\left[0, t^{*}\right) .
\end{aligned}
$$

Case I. Let $t^{*}>\tau\left(t^{*}\right)>0$. We have

$$
\begin{aligned}
\left\|x\left(t^{*}\right)\right\| & \leq|p|\left\|x\left(t^{*}-\tau\left(t^{*}\right)\right)\right\|+M e^{-\mu t^{*}} \\
& \leq|p|\left\{\left\|x_{0}\right\|_{s} e^{-m\left(t^{*}-\tau\left(t^{*}\right)\right)}+\frac{M}{1-|p| e^{\epsilon \tau}} e^{-\epsilon\left(t^{*}-\tau\left(t^{*}\right)\right)}\right\}+M e^{-\epsilon t^{*}}
\end{aligned}
$$




$$
\begin{aligned}
& \leq|p| e^{m \tau}\left\|x_{0}\right\|_{s} e^{-m t^{*}}+\frac{M|p| e^{\epsilon \tau}}{1-|p| e^{\epsilon \tau}} e^{-\epsilon t^{*}}+M e^{-\epsilon t^{*}} \\
& \leq\left\|x_{0}\right\|_{s} e^{-m t^{*}}+\frac{M}{1-|p| e^{\epsilon \tau}} e^{-\epsilon t^{*}} \equiv N e^{-\varphi t^{*}} .
\end{aligned}
$$

Case II. Let $-\tau<0<t^{*}<\tau\left(t^{*}\right)$. Then $\left\|x\left(t^{*}-\tau\left(t^{*}\right)\right)\right\| \leq\left\|x_{0}\right\|_{s}=\sup _{s \in[-\tau, 0]}\|x(s)\|$; thus,

$$
\begin{aligned}
\left\|x\left(t^{*}\right)\right\| & \leq|p|\left\|x\left(t^{*}-\tau\left(t^{*}\right)\right)\right\|+M e^{-\mu t^{*}} \\
& \leq\left\|x_{0}\right\|_{s} e^{-m t^{*}}+\frac{M}{1-|p| e^{\epsilon \tau}} e^{-\epsilon t^{*}} \equiv N e^{-\varphi t^{*}} .
\end{aligned}
$$

For both cases, there is contradiction to the inequality (7). Therefore, the inequality (6) holds for all $t \geq 0$.

\section{Main results}

In this section, the globally exponential stability for the NDE with time-varying delays in equation (1) will be presented as follows.

Theorem 4 For given $a>0, b>0, k>0$ and $\tau(t), \sigma(t)$ satisfying (2), the neutral differential equation (1) is globally exponentially stable if the operator $D$ is stable (i.e. $|p|<1)$ and there exist positive scalars $q_{1}, \alpha, \beta$, and scalars $q_{i}, i=2,3, \ldots, 6$ such that

$$
\Omega=\left[\begin{array}{ccccc}
2 k q_{1}-2 q_{2} & (1,2) & q_{2} p+q_{3} & b q_{1} & q_{3} \\
* & (2,2) & q_{4} p+q_{5}+2 q_{6} & 0 & q_{5}+2 q_{6} \\
* & * & (3,3) & 0 & -2 q_{6} \\
* & * & * & -\beta\left(1-\delta_{2}\right) e^{-2 k \sigma} & 0 \\
* & * & * & * & -2 q_{6}
\end{array}\right]<0,
$$

where $(1,2)=-a q_{1}+q_{2}-q_{3}-q_{4},(2,2)=2 q_{4}-2 q_{5}-2 q_{6}+\alpha+\beta,(3,3)=-2 q_{6}-\alpha\left(1-\delta_{1}\right) e^{-2 k \tau}$.

Proof Choose a Lyapunov-Krasovskii functional candidate $V=V_{1}+V_{2}+V_{3}$ where

$$
\begin{aligned}
& V_{1}(t)=e^{2 k t}\left[\begin{array}{lll}
D & x(t) & 0
\end{array}\right]\left[\begin{array}{lll}
1 & 0 & 0 \\
0 & 0 & 0 \\
0 & 0 & 0
\end{array}\right]\left[\begin{array}{ccc}
q_{1} & 0 & 0 \\
q_{2} & q_{3} & 0 \\
q_{4} & q_{5} & q_{6}
\end{array}\right]\left[\begin{array}{c}
D \\
x(t) \\
0
\end{array}\right]=e^{2 k t} q_{1} D^{2}, \\
& V_{2}(t)=\alpha \int_{t-\tau(t)}^{t} e^{2 k s} x^{2}(s) d s+\beta \int_{t-\sigma(t)}^{t} e^{2 k s} \tanh ^{2} x(s) d s, \\
& V_{3}(t)=\gamma e^{2 k t} D^{2}
\end{aligned}
$$

where $D=x(t)+p x(t-\tau(t)), q_{1}>0, \alpha>0, \beta>0, q_{i}, i=2,3, \ldots, 6$ are real numbers and $\gamma$ is a positive number that will be determined later.

Taking derivatives of $V_{1}(t)$ and $V_{2}(t)$ along the trajectory of equation (4), we have

$$
\begin{aligned}
\dot{V}_{1}(t) & =e^{2 k t}\left[2 k q_{1} D^{2}+2 q_{1} D \dot{D}\right] \\
& =2 e^{2 k t} k q_{1} D^{2}+2 e^{2 k t}\left[\begin{array}{lll}
D & x(t) & 0
\end{array}\right]\left[\begin{array}{ccc}
q_{1} & q_{2} & q_{3} \\
0 & q_{4} & q_{5} \\
0 & 0 & q_{6}
\end{array}\right]\left[\begin{array}{c}
\dot{D} \\
0 \\
0
\end{array}\right] .
\end{aligned}
$$


Utilizing the Leibniz-Newton formula, $\int_{t-\tau(t)}^{t} \dot{x}(s) d s=x(t)-x(t-\tau(t))$, we obtain

$$
\begin{aligned}
\dot{V}_{1}(t)= & 2 e^{2 k t} k q_{1} D^{2} \\
& +2 e^{2 k t}\left[\begin{array}{lll}
D & x(t) & -\int_{t-\tau(t)}^{t} \dot{x}(s) d s+x(t)-x(t-\tau(t))
\end{array}\right]\left[\begin{array}{ccc}
q_{1} & q_{2} & q_{3} \\
0 & q_{4} & q_{5} \\
0 & 0 & q_{6}
\end{array}\right] \\
& \times\left[\begin{array}{c}
-a x(t)+b \tanh x(t-\sigma(t)) \\
-D+x(t)+p x(t-\tau(t))
\end{array}\right] \\
= & e^{2 k t}\left\{\begin{array}{c}
\left(2 k q_{1}-2 q_{2}\right) D^{2}+2\left(-a q_{1}+q_{2}-q_{3}-q_{4}\right) x(t) D \\
+ \\
+
\end{array}\right] 2\left(q_{4}-q_{5}-q_{6}\right) x^{2}(t)+2 b q_{1} D \tanh x(t-\sigma(t)) \\
& +2\left(q_{2} p+q_{3}\right) D x(t-\tau(t))+2\left(q_{4} p+q_{5}+2 q_{6}\right) x(t) x(t-\tau(t)) \\
& +2 q_{3} D \int_{t-\tau(t)}^{t} \dot{x}(s) d s-2 q_{6} x^{2}(t-\tau(t))-4 q_{6} x(t-\tau(t)) \int_{t-\tau(t)}^{t} \dot{x}(s) d s \\
& \left.+2\left(q_{5}+2 q_{6}\right) x(t) \int_{t-\tau(t)}^{t} \dot{x}(s) d s-2 q_{6}\left(\int_{t-\tau(t)}^{t} \dot{x}(s) d s\right)^{2}\right\}, \\
\dot{V}_{2}(t)= & \alpha e^{2 k t} x^{2}(t)-\alpha e^{2 k(t-\tau(t))}(1-\dot{\tau}(t)) x^{2}(t-\tau(t)) \\
& +\beta e^{2 k t} \tanh ^{2} x(t)-\beta e^{2 k(t-\sigma(t))}(1-\dot{\sigma}(t)) \tanh ^{2} x(t-\sigma(t)) .
\end{aligned}
$$

Since $\tau(t) \leq \tau, \sigma(t) \leq \sigma, \dot{\tau}(t)<\delta_{1}, \dot{\sigma}(t)<\delta_{2}$ and $\tanh ^{2} x(t) \leq x^{2}(t)$, we have

$$
\begin{gathered}
\dot{V}_{2}(t) \leq e^{2 k t}\left\{(\alpha+\beta) x^{2}(t)-\alpha e^{-2 k \tau}\left(1-\delta_{1}\right) x^{2}(t-\tau(t))\right. \\
\left.-\beta e^{-2 k \sigma}\left(1-\delta_{2}\right) \tanh ^{2} x(t-\sigma(t))\right\} .
\end{gathered}
$$

Combining equations (9) and (10), we have

$$
\begin{aligned}
\dot{V}_{1}(t)+\dot{V}_{2}(t) \leq & e^{2 k t}\left\{\left(2 k q_{1}-2 q_{2}\right) D^{2}+2\left(-a q_{1}+q_{2}-q_{3}-q_{4}\right) x(t) D\right. \\
& +2\left(q_{4}-q_{5}-q_{6}+\alpha+\beta\right) x^{2}(t)+2 b q_{1} D \tanh x(t-\sigma(t)) \\
& +2\left(q_{2} p+q_{3}\right) D x(t-\tau(t))+2\left(q_{4} p+q_{5}+2 q_{6}\right) x(t) x(t-\tau(t)) \\
& +2 q_{3} D \int_{t-\tau(t)}^{t} \dot{x}(s) d s+2\left(q_{5}+2 q_{6}\right) x(t) \int_{t-\tau(t)}^{t} \dot{x}(s) d s \\
& +\left(-2 q_{6}-\alpha\left(1-\delta_{1}\right) e^{-2 k \tau}\right) x^{2}(t-\tau(t))-4 q_{6} x(t-\tau(t)) \int_{t-\tau(t)}^{t} \dot{x}(s) d s \\
& \left.-\beta\left(1-\delta_{2}\right) e^{-2 k \sigma} \tanh ^{2} x(t-\sigma(t))-2 q_{6}\left(\int_{t-\tau(t)}^{t} \dot{x}(s) d s\right)^{2}\right\} \\
= & e^{2 k t} \ell^{T}(t) \Omega \ell(t),
\end{aligned}
$$

where $\ell(t)=\left[D, x(t), x(t-\tau(t)), \tanh x(t-\sigma(t)), \int_{t-\tau(t)}^{t} \dot{x}(s) d s\right]^{T}$ and $\Omega$ is defined in equation (8). Since $\Omega<0$, we have $\dot{V}_{1}(t)+\dot{V}_{2}(t) \leq e^{2 k t} \ell^{T}(t) \Omega \ell(t)<0$. Therefore, there is a positive 
number $\lambda$ such that

$$
\begin{aligned}
\dot{V}_{1}(t)+\dot{V}_{2}(t) \leq & -\lambda e^{2 k t}\left(\|D\|^{2}+\|x(t)\|^{2}+\|x(t-\tau(t))\|^{2}+\|\tanh x(t-\sigma(t))\|^{2}\right. \\
& \left.+\left\|\int_{t-\tau(t)}^{t} \dot{x}(s) d s\right\|^{2}\right) \\
\leq & -\lambda e^{2 k t}\|x(t)\|^{2} .
\end{aligned}
$$

Taking the derivative of $V_{3}$ along the trajectory of equation (4) and utilizing the Cauchy inequality (Lemma 2), we have

$$
\begin{aligned}
\dot{V}_{3}(t)= & 2 e^{2 k t} \gamma\left(D \dot{D}+k D^{2}\right) \\
= & 2 e^{2 k t} \gamma\{(x(t)+p x(t-\tau(t)))(-a x(t)+b \tanh x(t-\sigma(t))) \\
& \left.+k(x(t)+p x(t-\tau(t)))^{2}\right\} \\
\leq & e^{2 k t} \gamma\left\{\left(2 k-2 a+4|p k|^{2}+|a p|^{2}+|b|^{2}\right) x^{2}(t)+\left(2 p^{2} k+2+|p b|^{2}\right) x^{2}(t-\tau(t))\right. \\
& \left.+2 \tanh ^{2} x(t-\sigma(t))\right\} .
\end{aligned}
$$

We choose

$$
\gamma=\left\{\begin{array}{lr}
\frac{\lambda}{2} \min \left\{\frac{1}{2 p^{2} k+|p b|^{2}+2}, \frac{1}{2}\right\}, & \text { if } 2 k-2 a+4|p k|^{2} \\
\frac{\lambda}{2} \min \left\{\frac{1}{2 k-2 a+4|p k|^{2}+|a p|^{2}+|b|^{2}}, \frac{1}{2 p^{2} k+|p b|^{2}+2}, \frac{1}{2}\right\}, & \text { otherwise. }
\end{array}\right.
$$

We obtain $\dot{V}(t)=\dot{V}_{1}(t)+\dot{V}_{2}(t)+\dot{V}_{3}(t) \leq-\frac{\lambda}{2} e^{2 k t}\|x(t)\|^{2}<0$. From the condition that $\dot{V}(t)$ is negative definite and $0 \leq \tau(t) \leq \tau, 0 \leq \sigma(t) \leq \sigma$, we have $V(x(t)) \leq V(x(0))$, for all $t \geq 0$, with

$$
\begin{aligned}
V(x(0))= & V_{1}(x(0))+V_{2}(x(0))+V_{3}(x(0)) \\
= & q_{1}(x(0)+p x(-\tau(0)))^{2}+\alpha \int_{-\tau(0)}^{0} e^{2 k s} x^{2}(s) d s \\
& +\beta \int_{-\sigma(0)}^{0} e^{2 k s} \tanh ^{2}(x(s)) d s+\gamma(x(0)+p x(-\tau(0)))^{2} \\
\leq & q_{1}(1+|p|)^{2}\left\|x_{0}\right\|_{s}^{2}+\alpha \int_{-\tau(0)}^{0} e^{2 k s}\left(\sup _{-r \leq s \leq 0}\|x(s)\|\right)^{2} d s \\
& +\beta \int_{-\sigma(0)}^{0} e^{2 k s}\left(\sup _{-r \leq s \leq 0}\|x(s)\|\right)^{2} d s+\gamma(1+|p|)^{2}\left\|x_{0}\right\|_{s}^{2} \\
\leq & q_{1}(1+|p|)^{2}\left\|x_{0}\right\|_{s}^{2}+\alpha \tau\left\|x_{0}\right\|_{s}^{2}+\beta \sigma\left\|x_{0}\right\|_{s}^{2}+\gamma(1+|p|)^{2}\left\|x_{0}\right\|_{s}^{2} \\
= & \Delta\left\|x_{0}\right\|_{s}^{2},
\end{aligned}
$$

where $\Delta=q_{1}(1+|p|)^{2}+\alpha \tau+\beta \sigma+\gamma(1+|p|)^{2}$.

From $\gamma e^{2 k t}\|D\|^{2} \leq V(x(t)) \leq \Delta\left\|x_{0}\right\|_{s}^{2}$, we obtain $\|D\| \leq M e^{-k t}$ where $M=\sqrt{\frac{\Delta}{\gamma}}\left\|x_{0}\right\|_{s}$. Because $D=x(t)+p x(t-\tau(t))$, we have

$$
\|x(t)\|=\|D-p x(t-\tau(t))\| \leq\|D\|+|p|\|x(t-\tau(t))\| \leq M e^{-k t}+|p|\|x(t-\tau(t))\| .
$$


Since $|p|<1$ and $0 \leq \tau(t) \leq \tau$, we can choose a sufficiently small positive constant $\varphi=k<$ $\frac{-\ln |p|}{\tau}$ so that $|p| e^{\varphi \tau}<1$. Utilizing Proposition 3, we have

$$
\|x(t)\| \leq\left(\left\|x_{0}\right\|_{s}+\frac{M}{1-|p| e^{\varphi \tau}}\right) e^{-\varphi t}, \quad t \geq 0 .
$$

Choosing $\mu=\max \left\{\left\|x_{0}\right\|_{s}, \frac{M}{1-|p| e^{\varphi \tau}}\right\}$, we obtain

$$
\|x(t)\| \leq 2 \mu e^{-\varphi t}
$$

This implies that the zero solution of equation (1) is exponentially stable. By radially unboundedness, it is globally exponentially stable with a rate of convergence $k=\varphi>0$.

Remark 5 If $k=0$, one can easily see that the zero solution of equation (1) is uniformly asymptotically stable when the following criterion holds:

$$
\Omega=\left[\begin{array}{ccccc}
-2 q_{2} & (1,2) & q_{2} p+q_{3} & b q_{1} & q_{3} \\
* & (2,2) & q_{4} p+q_{5}+2 q_{6} & 0 & q_{5}+2 q_{6} \\
* & * & -2 q_{6}-\alpha\left(1-\delta_{1}\right) & 0 & -2 q_{6} \\
* & * & * & -\beta\left(1-\delta_{2}\right) & 0 \\
* & * & * & * & -2 q_{6}
\end{array}\right]<0,
$$

where $(1,2)=-a q_{1}+q_{2}-q_{3}-q_{4},(2,2)=2 q_{4}-2 q_{5}-2 q_{6}+\alpha+\beta$.

Remark 6 Our globally exponential stability criterion (8) is delay dependent relating to delays $\tau, \sigma$ but the uniformly asymptotic stability criterion (11) is delay independent. In addition, both criteria depend on the rates of change of the delays $\delta_{1}$ and $\delta_{2}$. These criteria are found to be less conservative than those in $[7,8]$ because our rate of change of delay $\delta_{1}$ does not require it to be less than 1 but it is so in $[7,8]$.

\section{Numerical examples}

In this section, four numerical examples are given to show the effectiveness of our main results by comparing the upper bounds of the delays $\sigma$ and the parameter $b$ as well as investigating the rate of convergence. The feasibility of all criteria are obtained by using the LMI control toolbox in MATLAB.

Example 1 Consider the following equation with time-varying delays:

$$
\frac{d}{d t}[x(t)+0.1 x(t-\tau(t))]=-1.5 x(t)+0.2 \tanh x(t-\sigma(t)), \quad t \geq 0,
$$

when $\tau(t)=2 \sin ^{2}(t)$ and $\sigma(t)=\frac{\cos ^{2}(t)}{5}$.

Solving the condition (8), we obtain a set of parameters guaranteeing globally exponential stability as follows:

$$
\begin{aligned}
& k=0.6479, \quad \delta_{1}=0.0371, \quad \delta_{2}=0.0371, \\
& \alpha=0.8492, \quad \beta=1.0068, \quad q_{1}=1.4631,
\end{aligned}
$$




$$
\begin{aligned}
& q_{2}=1.0694, \quad q_{3}=0.0369, \quad q_{4}=-1.3427, \\
& q_{5}=-0.1263, \quad q_{6}=0.1414 .
\end{aligned}
$$

Moreover, we investigate the feasibility of the criterion (8) by varying the values of parameter $p$ and find that the criterion maintains its feasibility up to $p=0.6$. This example shows that our proposed condition (8) is practical for the NDE with time-varying delays.

Example 2 Consider the following equation studied in $[7,8]$ :

$$
\frac{d}{d t}[x(t)+0.2 x(t-\tau(t))]=-0.6 x(t)+0.5 \tanh x(t-\sigma(t)), \quad t \geq 0,
$$

when $\tau(t)=\frac{\sin ^{2}(t)}{10}$ and $\delta_{2}=0.2$.

Solving our criterion (11), guaranteeing a uniformly asymptotic stability, allows an upper bound $\sigma=10^{25}$, which is fairly the same as found in other existing work [7, 8].

In case of exponential stability, when $k=0.0038$ is given, our criterion (8) allows the upper bound of the time delay $\sigma(t)=7.5231$ along with a set of parameters guaranteeing globally exponential stability as follows:

$$
\begin{aligned}
& \alpha=0.0002, \quad \delta_{1}=0.0058, \quad \beta=0.0056, \quad q_{1}=0.0097, \quad q_{2}=0.0058, \\
& q_{3}=3.0232 \times e^{-8}, \quad q_{4}=-0.0058, \quad q_{5}=-3.139 \times e^{-8}, \quad q_{6}=4212.86 .
\end{aligned}
$$

Furthermore, we vary the rate of convergence and find that our criterion (8) is still feasible up to $k=0.028$ along with the upper bound $\sigma(t)=0.0321$. However, the criterion proposed in [8] is only feasible for an asymptotically stable case, while the criterion in [7] gives no information on $k$ (see the comparison in Table 1). This example shows that our criterion (8) is less conservative than previous results.

Example 3 Consider the following equation from $[7-9,12,13,15,16]$ :

$$
\frac{d}{d t}[x(t)+0.2 x(t-0.1)]=-0.6 x(t)+0.3 \tanh x(t-\sigma), \quad t \geq 0 .
$$

By solving LMI (11), it allows the upper bound of the time delay $\sigma=10^{21}$, which guarantees uniformly asymptotic stability. When $k=0.0038$ is given, solving LMI (8) yields the upper bound of the time delay $\sigma=175.354$ along with a set of parameters guaranteeing globally exponential stability as follows:

$$
\begin{aligned}
& \alpha=0.0002, \quad \beta=0.0041, \quad q_{1}=0.0072, \quad q_{2}=0.0043, \\
& q_{3}=-4.6203 \times 10^{-11}, \quad q_{4}=-0.0043, \quad q_{5}=5.5829 \times 10^{-11}, \quad q_{6}=1.1878 .
\end{aligned}
$$

Table 1 Bound on time delay $\sigma(t)$ for ensuring stability of equation (1)

\begin{tabular}{llllll}
\hline & & [8] & [7] & Our equation (8) & Our equation (11) \\
\hline A.S. & & $10^{25}$ & $10^{24}$ & - & $10^{25}$ \\
E.S. & $k=0.0038$ & infeasible & - & 7.5231 & - \\
& $k=0.02$ & infeasible & - & 0.5234 & - \\
& $k=0.028$ & infeasible & - & 0.0321 & - \\
\hline
\end{tabular}


Table 2 Bound on time delay $\sigma$ for ensuring stability of equation (3) in Example 3

\begin{tabular}{llllllllll}
\hline & [8] & [7] & [9] & [12] & [13] & [15] & [16] & Our equation (8) & Our equation (11) \\
\hline A.S. & $10^{21}$ & - & 2.22 & $10^{7}$ & 2.32 & 1.902 & 2.32 & - & $10^{21}$ \\
E.S. & 175.289 & $10^{21}$ & - & - & - & - & 1.947 & 175.354 & - \\
$(k=0.0038)$ & & $($ No $k)$ & & & & & & & \\
\hline
\end{tabular}

Notations: 'A.S.' and 'E.S.' stand for asymptotically and exponentially stable, respectively. 'No $k$ ' means that the rate of convergence cannot be specified.

Table 3 Bound on $b$ for ensuring stability of equation (3) in Example 4

\begin{tabular}{llllllllll}
\hline & [8] & [7] & [9] & [18] & [13] & [15] & [16] & Our equation (8) & Our equation (11) \\
\hline A.S. & 1.405 & - & 0.899 & 0.722 & 1.405 & 0.699 & 1.405 & - & 1.405 \\
E.S. & 1.0929 & 1.346 & - & - & - & - & 0.478 & 1.1089 & - \\
$(k=0.177)$ & & $(\mathrm{Nok})$ & & & & & & & \\
\hline
\end{tabular}

In Table 2, we compare the upper bounds of $\sigma$ with other work. It is clear that the upper bounds from our criteria (8) and (11) are larger than many values obtained from other work.

Example 4 Consider the following equation, studied in $[7-9,13,15,16,18]$ :

$$
\frac{d}{d t}[x(t)+0.35 x(t-0.5)]=-1.5 x(t)+b \tanh x(t-0.5), \quad t \geq 0 .
$$

By solving LMI (11), it allows the upper bound of the parameter $b=1.405$, ensuring uniformly asymptotic stability. When $k=0.177$ is given, solving LMI (8) allows the upper bound $b=1.1089$, with a set of parameters guaranteeing the exponential stability as follows:

$$
\begin{aligned}
& b=1.1089, \quad \alpha=4.1038 \times 10^{-5}, \quad \beta=0.0002, \\
& q_{1}=0.0002, \quad q_{2}=0.0003, \quad q_{3}=6.0599 \times e^{-12}, \\
& q_{4}=-0.0003, \quad q_{5}=-2.5512 \times e^{-12}, \quad q_{6}=6.2325 .
\end{aligned}
$$

The values of the upper bounds $b$ are compared with other work (see Table 3 ). The results show that our upper bound $b$ is larger than the values obtained from other work.

Furthermore, we have investigated the rate of convergence by fixing the value of $b=$ 1.346 (as obtained by [7]), and solving criterion (8) yields the rate of convergence of $k=$ 0.0343 , while no information on $k$ can be specified from the criterion in [7].

Remark 7 One can obtain a faster speed of convergence up to $k=0.2489$ in Example 3 and up to $k=0.93$ in Example 4 with trade-off in smaller upper bounds decreasing to near zero.

Remark 8 It is worth pointing out that the upper bounds from [7] are larger than the values obtained by solving our LMI (8) as seen in Tables 2 and 3. However, the upper bounds in [7] are given without any information of the rate of convergence.

\section{Conclusion}

In this paper, we have proved an important inequality associated with a time-varying neutral delay. Then we proposed two criteria for ensuring globally exponential stability and 
uniformly asymptotic stability of the neutral differential equation with time-varying delays. Finally, four numerical examples are given to show that the proposed criteria are less conservative than much existing work.

\section{Competing interests}

All authors declare that they have no competing interests.

\section{Authors' contributions}

The work presented here was carried out in collaboration between both authors. TR designed the problem and initiated techniques used in this work. Both authors carried out the analysis. PK ran numerical tests. TR wrote the manuscript and both authors read and approved the final manuscript.

\section{Acknowledgements}

The authors would like to thank the anonymous referees for their valuable comments and suggestions, which have led to significant improvement of this paper. This research is fully supported by Chiang Mai University.

Received: 18 September 2013 Accepted: 8 January 2014 Published: 27 Jan 2014

\section{References}

1. Fridman, E: Stability of linear descriptor systems with delays: a Lyapunov-based approach. J. Math. Anal. Appl. 273 24-44 (2002)

2. Kwon, OM, Park, J, Lee, SM: Augmented Lyapunov functional approach to stability of uncertain neutral systems with time-varying delays. Appl. Math. Comput. 207, 202-212 (2009)

3. Liao, X, Liu, Y, Guo, S, Mai, H: Asymptotic stability of delayed neural networks: a descriptor system approach. Commun. Nonlinear Sci. Numer. Simul. 14, 3120-3133 (2009)

4. Logemann, H, Twonley, S: The effects of small delays in the feedback loop on the stability of the neutral systems. Syst. Control Lett. 27, 267-274 (1996)

5. Park, JH: Delay-dependent criterion for guaranteed cost control of neutral delay systems. J. Optim. Theory Appl. 124, 491-502 (2005)

6. Agarwal, RP, Grace, SR: Asymptotic stability of certain neutral differential equations. Math. Comput. Model. 31, 9-15 (2000)

7. Chen, $H$, Meng, $X$ : An improved exponential stability criterion for a class of neutral delayed differential equations. Appl. Math. Lett. 24,1763-1767 (2011)

8. Chen, H: Some improved criteria on exponential stability of neutral differential equation. Adv. Differ. Equ. 2012, 170 (2012). doi:10.1186/1687-1847-2012-170

9. Deng, S, Liao, X, Guo, S: Asymptotic stability analysis of certain neutral differential equations: a descriptor system approach. Math. Comput. Simul. 71, 4297-4308 (2009)

10. El-Morshedy, HA, Gopalsamy, K: Nonoscillation, oscillation and convergence of a class of neutral equations. Nonlinear Anal. 40, 173-183 (2000)

11. Hale, JK, Verduyn Lunel, SM: Introduction to Functional Differential Equations. Springer, New York (1993)

12. Kwon, OM, Park, JH: On improved delay-dependent stability criterion of certain neutral differential equations. Appl. Math. Comput. 199, 385-391 (2008)

13. Nam, PT, Phat, VN: An improved stability criterion for a class of neutral differential equations. Appl. Math. Lett. 22, 31-35 (2009)

14. Park, JH: Delay-dependent criterion for asymptotic stability of a class of neutral equations. Appl. Math. Lett. 17, 1203-1206 (2004)

15. Park, JH, Kwon, OM: Stability analysis of certain nonlinear differential equation. Chaos Solitons Fractals $27,450-453$ (2008)

16. Rojsiraphisal, T, Niamsup, P: Exponential stability of certain neutral differential equations. Appl. Math. Lett. 17, 3875-3880 (2010)

17. Sun, YG, Wang, L: Note on asymptotic stability of a class of neutral differential equations. Appl. Math. Lett. 19, 949-953 (2006)

18. Li, X: Global exponential stability for a class of neural networks. Appl. Math. Lett. 22, 1235-1239 (2009)

19. Fridman, E: New Lyapunov-Krasovskii functionals for stability of linear retarded and neutral type systems. Syst. Control Lett. 43, 309-319 (2001)

10.1186/1687-1847-2014-32

Cite this article as: Keadnarmol and Rojsiraphisal: Globally exponential stability of a certain neutral differential equation with time-varying delays. Advances in Difference Equations 2014, 2014:32 